\title{
A FAMILY OF POLYNOMIALS WITH CONCYCLIC ZEROS
}

\author{
KENNETH B. STOLARSKY
}

ABstract. Expand $E(z)=\left(e^{z}-1\right)^{m}$ by the binomial theorem, and replace every $\exp (k z)$ by its approximation $\left(1+k n^{-1} z\right)^{n}$. The resulting polynomial has all of its zeros on a circle of radius $r$ centered at $-r$, where $r=n / m$.

1. Introduction. For positive integers $n$ let $P_{n}$ be the linear mapping from the exponential polynomials over $\mathbf{C}$ to the polynomials over $\mathbf{C}$ that replaces $\exp (a z)$ by

$$
\left(1+\frac{a z}{n}\right)^{n}
$$

but is otherwise the identity. For example,

$$
P_{n}\left\{\left(e^{z}-1\right)^{2}\right\}=\left(1+\frac{2 z}{n}\right)^{n}-2\left(1+\frac{z}{n}\right)^{n}+1 .
$$

Thus $P_{\infty}$ applied to any exponential polynomial $E(z)$ would be the identity.

Our purpose is to establish Conjecture 2 of [14]. This is now

THEOREM 1. Let $n$ and $m$ be positive integers with $n \geqslant m$. The zeros of

$$
P_{n}\left\{\left(e^{z}-1\right)^{m}\right\}=\left(1+\frac{m z}{n}\right)^{n}-\cdots
$$

all lie on $C(r)$, the circle of radius $r$ centered at $-r$, where $r=n / m$.

Set

$$
w=1+\frac{m z}{n} .
$$

It suffices to show that $w$ or $w^{-1}$ lies on the unit circle $U$. But this follows from the binomial expansion and Theorem 3 .

The proof of Theorem 3 relies on exploiting a certain linear fractional transformation and upon

Theorem 2 (A. COHN, 1922). If $P(w)$ is a real polynomial of degree $n$ and

$$
w^{n} P\left(w^{-1}\right)= \pm P(w),
$$

then the zeros of $P$ all lie on $U$ if and only if the zeros of its derivative all lie on or inside $U$.

For Theorem 2 (and a bit more) see [3 or 6, p. 206].

Received by the editors October 25, 1982.

1980 Mathematics Subject Classification. Primary 30C15; Secondary 33A10.

Key words and phrases. Concyclic zeros, exponential, exponential polynomial, linear fractional transformation, "reductionism", zeros of polynomials. 
2. The proof. We now establish

THEOREM 3. Let $n \geqslant m$. Then the polynomial

$$
R_{m}(w)=\sum_{k=0}^{m}(-1)^{k}\left(\begin{array}{c}
m \\
k
\end{array}\right)[k w+(m-k)]^{n}
$$

has all its roots on $U$.

Proof. It is clear for $m=1$. Assume the result for $m-1$. Since

$$
R_{m}^{\prime}(w)=n \sum_{k=0}^{m}(-1)^{k}\left(\begin{array}{c}
m \\
k
\end{array}\right) k[k w+(m-k)]^{n-1}
$$

it suffices by A. Cohn's theorem to show that the zeros of

$$
S_{m}(w)=\sum_{k=1}^{m}(-1)^{k}\left(\begin{array}{c}
m \\
k
\end{array}\right) \frac{k}{m}[k w+(m-k)]^{n-1}
$$

have modulus at most 1 . Let

$$
z=\frac{m w}{w+m-1} \quad \text { so } w=\frac{(m-1) z}{m-z} .
$$

Then

$$
k w+(m-k)=m \cdot \frac{(k-1) z+m-k}{m-z}
$$

so

$$
\begin{aligned}
\left(\frac{m-z}{m}\right)^{n-1} S_{m}(w) & =\sum_{k=1}^{m}(-1)^{k}\left(\begin{array}{c}
m-1 \\
k-1
\end{array}\right)[(k-1) z+m-k]^{n-1} \\
& =-\sum_{k=0}^{m-1}(-1)^{k}\left(\begin{array}{c}
m-1 \\
k
\end{array}\right)[k z+(m-1-k)]^{n-1}
\end{aligned}
$$

By the induction hypothesis, the vanishing of $S_{m}(w)$ implies that $z$ is on $U$. Hence

$$
|w| \leqslant \frac{(m-1)|z|}{m-|z|}=1
$$

and the result follows.

We remark that by making further linear fractional transformations one can show that the roots of

$$
\sum_{k=0}^{m}(-1)^{k}\left(\begin{array}{c}
m \\
k
\end{array}\right)[(k-1) z-(k-2)]^{n}=0
$$

lie on a circle about $(m-3) /(m-2)$ of radius $1 /(m-2)$, and that for any positive integer $h$ the roots of

$$
\sum_{k=0}^{m}(-1)^{k}\left(\begin{array}{c}
m \\
k
\end{array}\right)[(k-h) z+(m-k-h)]^{n}
$$

lie on $U$. For the study of a more difficult family of polynomials of which (2.1) is a special case when $m=2$, see [7]. 
REMARKS. The paper [14] shows how this fits into the general program of "reductionism". The author's previous proof of the theorem (for $m=4$ only) involved the much heavier machinery of [7]. For further material on zeros of exponential polynomials see, e.g., [1, pp. 120-122], [2, 4, 5, 8, 9, 12, 15], and the many references therein.

For another way in which linear fractional transformations enter into proofs of similar results, see [10] and the idea of Dyson in [11].

In general, if $E(z)$ is an exponential polynomial with all zeros real, then the zeros of $P_{n} E(z)$ lie in a narrow annulus; see [14].

ACKNOWLEDGEMENT. The author thanks Kevin McCurley for stimulating discussions and much assistance with computer generation of numerical examples.

\section{REFERENCES}

1. A. Baker, Transcendental number theory, Cambridge Univ. Press, Cambridge, 1975.

2. R. E. Bellman and K. L. Cooke, Differential-difference equations, Academic Press, New York, 1963.

3. A. Cohn, Über die Anzahl der Wurzeln einer algebraischen Gleichung in einem Kreise, Math. Z. 14 (1922), 110-148.

4. D. G. Dickson, Zeros of exponential sums, Proc. Amer. Math. Soc. 16 (1965), 84-89.

5. R. E. Langer, On the zeros of exponential sums and integrals, Bull. Amer. Math. Soc. 37 (1931), 213-239.

6. M. Marden, Geometry of polynomials, Math. Surveys, No. 3, Amer. Math. Soc., Providence, R.I. 1966.

7. J. D. Nulton and K. B. Stolarsky, The zeros of a certain family of trinomials, in preparation.

8. A. J. van der Poorten, On the number of zeros of functions, Enseign. Math. (2) 23 (1977), 19-38.

9. A. J. van der Poorten and R. Tijdeman, On common zeros of exponential polynomials, Enseign. Math. (2) 21 (1975), 57-67.

10. D. Ruelle, Some remarks on the location of zeros of the partition function for lattice systems, Comm. Math. Phys. 31 (1973), 265-277.

11. , Extension of the Lee-Yang circle theorem, Phys. Rev. Lett. 26 (1971), 303-304.

12. E. Schwengeler, Geometrisches über die Verteilung der Nullstellen spezieller ganzer funktionen, Thesis, Zürich, 1925.

13. K. B. Stolarsky, Zero-free regions for exponential sums, Proc. Amer. Math. Soc. 83 (1981), 486-488.

14. Z_ Zeros of exponential polynomials and "reductionism", Colloq. Math. Soc. János Bolyai, vol. 34, Topics in Classical Number Theory, Elsevier, New York; North-Holland, Amsterdam (to appear).

15. M. Voorhoeve, On the oscillation of exponential polynomials, Math. Z. 151 (1976), 277-294.

DePartment of Mathematics, 1409 West Green, University of Illinois, Urbana, Illinois 61801 